\title{
USING CITYGML TO DEPLOY SMART-CITY SERVICES FOR URBAN ECOSYSTEMS.
}

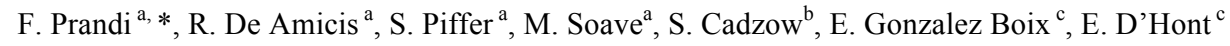 \\ ${ }^{a}$ Fondazione Graphitech, via alla cascata 56/C Trento, Italy - (federico.prandi, raffaele.de.amicis, stefano.piffer, \\ marco.soave)@graphitech.it \\ ${ }^{\mathrm{b}}$ Cadzow Communications Consulting Ltd., United Kingdom - scott@cadzow.com \\ ${ }^{\mathrm{c}}$ Vrije Universiteit Brussels, Artificial Intelligence Lab, BrusSense, Bruxelles, Belgium \\ - (egonzale, eldhondt)@vub.ac.be
}

Commission VI, WG VI/4

KEY WORDS: CityGML, 3D portrayal services, Visualization, Smart-Cities, Crowd Source,

\begin{abstract}
:
The rapid technological evolution, which is characterizing all the disciplines involved within the wide concept of smart cities, becomes a key factor to trigger true user-driven innovation. In this context $3 \mathrm{D}$ city models will play an increasingly important role in our daily lives and become an essential part of the modern city information infrastructure (Spatial Data Infrastructure). The goal of this paper is to introduce the i-SCOPE (interoperable Smart City services through an Open Platform for urban Ecosystems) project methodology and implementations together with key technologies and open standards. Based on interoperable 3D CityGML UIMs, the aim of i-Scope is to deliver an open platform on top of which it possible to develop, within different domains, various 'smart city' services.

Moreover, in i-SCOPE different issues, transcending the mere technological domain, are being tackled, including aspects dealing with social and environmental issues. Indeed several tasks including citizen awareness, crowd source and voluntary based data collection as well as privacy issue concerning involved people should be considered.
\end{abstract}

\section{INTRODUCTION}

\subsection{Motivation}

An increasing amount of people are living in cities and, by 2030, the number will be close to 5 billion (United Nations 2008). Therefore, it is essential to develop efficient techniques to assist the management of modern cities. The European Commission, within the so-called Digital Agenda, is paying significant attention to smart cities and, as technologies associated to smart cities can bring to an improved knowledgebased economy, to better social inclusion and, in more general term, to a more liveable environment.

The rapid technological evolution, which is characterizing all the disciplines involved within the wide concept of smart cities, becomes a key factor to trigger true user-driven innovation. In this context $3 \mathrm{D}$ city models will play an increasingly important role in our daily lives and become an essential part of the modern city information infrastructure (Spatial Data Infrastructure). Similar to the 2D cartographic maps, the 3D city models will be used to integrate various data from different sources for public accessible visualization and many other applications.

The latest generation of 3D Urban Information Models (UIM), created from accurate urban-scale geospatial information, can be used as basis to create smart web services based on geometric, semantic, morphological and structural information at urban scale level. CityGML (Open-GIS 2008) represents a very attractive solution that combines $3 \mathrm{D}$ information and semantic in-formation in a single data model.
The goal of this paper is to describe the i-SCOPE (interoperable Smart City services through an Open Platform for urban Ecosystems) project challenges and results together with key technologies and open standards. The aim of i-SCOPE is to deliver an open platform, based on interoperable 3D CityGML UIMs, on top of which it possible to deploy various 'smart city' services.

The main challenge of the work is to develop into the i-SCOPE framework an effective way to exploit the CityGML potentiality to provide Smart Cities services. Indeed while for whom to concern the $2 \mathrm{D}$, the capability to use maps and images as basis for several web-applications is well consolidated, provisioning of 3D Geographic Information via web is yet oriented to simple visualization with limited possibility to interact and exploit the semantic content of the dataset.

In this way the 3D City model handily enriched with the information to support specific services became the tools to visualize and better understand such phenomena at the city level. However-city itself is complex, and it is impossible for a standard to specify every detail of the city. For these reasons iSCOPE aims at providing a significant contribution in domain related to project smart city services, through to extension and wider adoption of CityGML as key enabling open standard for 3D smart city services. At this purpose, for each service specific application fields, implemented using the Application Domain Extensions (ADE), will be defined and proposed.

The smart services proposed address the following three scenarios:

- Improved inclusion and personal mobility of aging people and diversely able citizens;

\footnotetext{
* Corresponding author. This is useful to know for communication with the appropriate person in cases with more than one author.
} 
Energy dispersion \& solar energy potential assessment;

- $\quad$ Noise mapping \& simulation.

Moreover, in i-SCOPE different issues, transcending the mere technological domain, are being tackled, including aspects dealing with social and environmental issues. Indeed several tasks including citizen awareness, crowd source and voluntary based data collection as well as priva-cy issue concerning involved people should be considered. For this reasons an aim of the work is to create an open ecosystem made of public administrations, industries, SMEs, universities and research centres to capture user-driven requirements as well as the involvement of users and to promote fast uptake of project services and transfer of knowledge across stakeholders.

\subsection{State of the art}

The term Smart Cities has been adopted since 2005 by a number of technology companies (Cisco, 2005), (IBM, 2009) for the application of complex information systems for the application of complex information systems to integrate the operation of urban infrastructure and services such as buildings, transportation, electrical and water distribution, and public safety. Into (Ishida and Isbister) some similar terms such digital community, smart community, digital city, information city and e-city are discussed. However all of those alternative terms are used to refer a platform or a system of systems, essentially based on three components: Sensors, Networks and Engagement (actuators) (Roche and Abbas 2012).

Research on smart cities has been conducted by many organizations, such MIT Smart Cities Lab including SENSeable City Lab; the World Foundation for Smart Communities; URENIO research unit and the Lab for Global Information Networks, Unicamp and Limitless/Dubai World.

A smart city provides interoperable, Internet-based government services that enable ubiquitous connectivity to transform key government processes, both internally across departments and em-ployees both externally to citizens and businesses (A1Hader, et al 2009). A service-oriented archi-tecture (SOA) based on open standards; and innovative services ensures the needed flexibility to meet the requirements of governments and their users, citizens and businesses (Cook and Sajal 2005).

Location data is now commonly regarded as the fourth driver in the decision-making process. The location provides more intelligent data analysis due to improved analytical and visualisation capa-bilities.

The geographical dimension (space) of smart communities is varied; it can be extended from a city district up to a multimillion metropolis but generally not overcame the dimension of the city. Considering this dimension Georeferenced 3D models represent an increasingly accepted solution for storing and displaying information at urban scale (Döllner et al. 2006). As the 3D models be-come more accurate, more smart cities can be a human interface for new broadband network ser-vices (Ishida 2002). The main feature of these models is the capabilities to store all information of a city in a single data model, facilitating the use and the interoperability of the same by different agents. For this reason in this paper will be investigated the usage of a $3 \mathrm{D}$ city model to represent, handle and manage urban data in a smart city services platform. CityGML (OpenGIS 2008) repre-sents a very attractive solution that combines $3 \mathrm{D}$ information and semantic information in a single data model.

\section{I-SCOPE FRAMEWORK}

\subsection{CityGML as base services}

The 3D visualization provides operators, administrators and citizens with a reproduction of the city environment as much realistic as possible in order to improve information retrieval capabilities and to enhance the effectiveness of the smart services. In this way the 3D city model be-came the framework to aggregate a list of available free sources of information that are collected for some transactional purpose, such as road tolling, energy or water consumption billing. In order to achieve this result the concept of the 3D city model shift respect to the most recent and known 3D maps systems (McCarra, Darren 2012). These platforms are excellent in terms of visualization aspect and performance but they are not suitable for the smart city services deployment, indeed their capability to consider also the semantic aspect of the modelled object is quite limited. Apple 3D maps represent cities as a very dense and textured mesh while Google 3D buildings, available by Google maps and google earth platforms, are geometrically modelled using KML, which is not fully compliant for these purposes.

CityGML is a common information model for the representation of $3 \mathrm{D}$ urban objects. It is real-ised as an open data model and XML-based format for the storage and exchange of virtual 3D city models. As an OGC standard, CityGML plays a leading role in the modularisation of urban geospatial information.

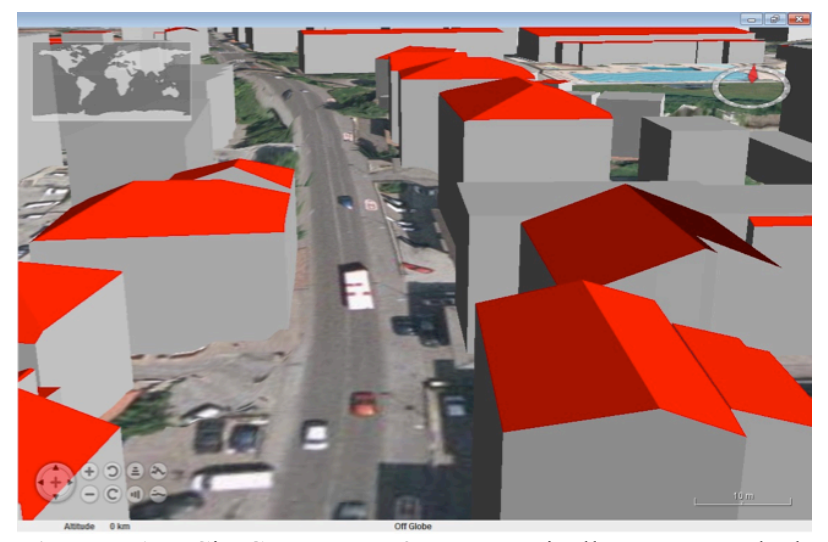

Figure 1: CityGML LoD2 automatically generated by novaFactory platform starting from buildings footprint and DSM

In the foreseeable future, 3D city models will play an increasingly important role in our daily lives and become an essential part of the modern city information infrastructure (Spatial Data Infrastructure). Similar to the 2D cartographic maps, the 3D city models will be used to inte-grate various data from different sources for public accessible visualisation and many other applications.

The main objective of the i-SCOPE project is to use the Urban Information Models (UIMs) en-coded into CityGML format as reference system for a series of smart services deployed in a so called "smart city" context. A main advantage of CityGML is a hierarchical geometrical and semantic structure, which allows to define each single component of the cities object (i.e. roof, wall, window) and associate it to the "father object" (i.e. building.). However despite the great advantages, CityGML is a quiet new data-model and it is no so spread at the local level. The most common situation, considering the i-SCOPE pilots, was that the municipalities could pro-vide at the disposal of the project this kind of information: 1) Digital Terrain Model (DTM), 2)Digital Surface Model (DSM) and 3) building footprint (2D). 
The first service developed into i-SCOPE project is a webservice to allowing the generation of 3D CityGML building objects into different LODs, starting from the abovementioned data (Fig.1). The footprint data allows identifying the area in which the DSM and DTM are analysed. A routine allows to identify whether cluster of points have a shape such as to identify a flat surface. These surfaces, identified within the area belonging to the footprint of a building, are compared with the planes composing some standard roofs' models. A good detection of the roofs composition is achieved having a minimum of 6 dots per roof area, therefore to recognize a gambled roof a minimum of 12 is necessary, meanwhile for a hipped roof a minimum of 24 points are needed.

The service is asynchronous and realized with novaFACTORY software solution, the user once finished the generation can validate, both downloading and visualizing the final result, both through a summary report the quality of the model.

After validation, CityGML data will be stored in 3DCityDB (2011), a free and Open Source 3D geo database to store, represent, and manage virtual 3D city models on top of the Oracle 10G R2 spatial (or 11G), developed by Institute for Geodesy and Geoinformation Science of the Ber-lin University of Technology.

The building model generated by i-SCOPE services is at the basis for the fruition of several smart-services focused on specific domain (energy, mobility, environment). Two main aspects have to be taken in account: 1) how to make the 3D

\subsection{CityGML extensions and ADEs}

CityGML make available data concerning the cities according to the open data model. The CityGML information model includes:

- Digital Terrain Models as a combination of triangulated irregular networks (TINs), regular raster, break and skeleton lines, mass points;

- $\quad$ Sites (buildings, bridges, tunnels...);

- Vegetation (areas, volumes, and solitary objects with vegetation classification);

- Water bodies (volumes and surfaces);

- Transportation facilities (both graph structures and 3D surface data);

- City furniture;

- $\quad$ Generic City objects and attributes;

Objects, which are not yet explicitly modelled in the current version of CityGML, can be represented using the concept of generic objects and attributes. Furthermore, the possibility of creating extensions to the standard pattern exists. This allows the description of object and proprieties not included in the standard characterized by specific properties and features.

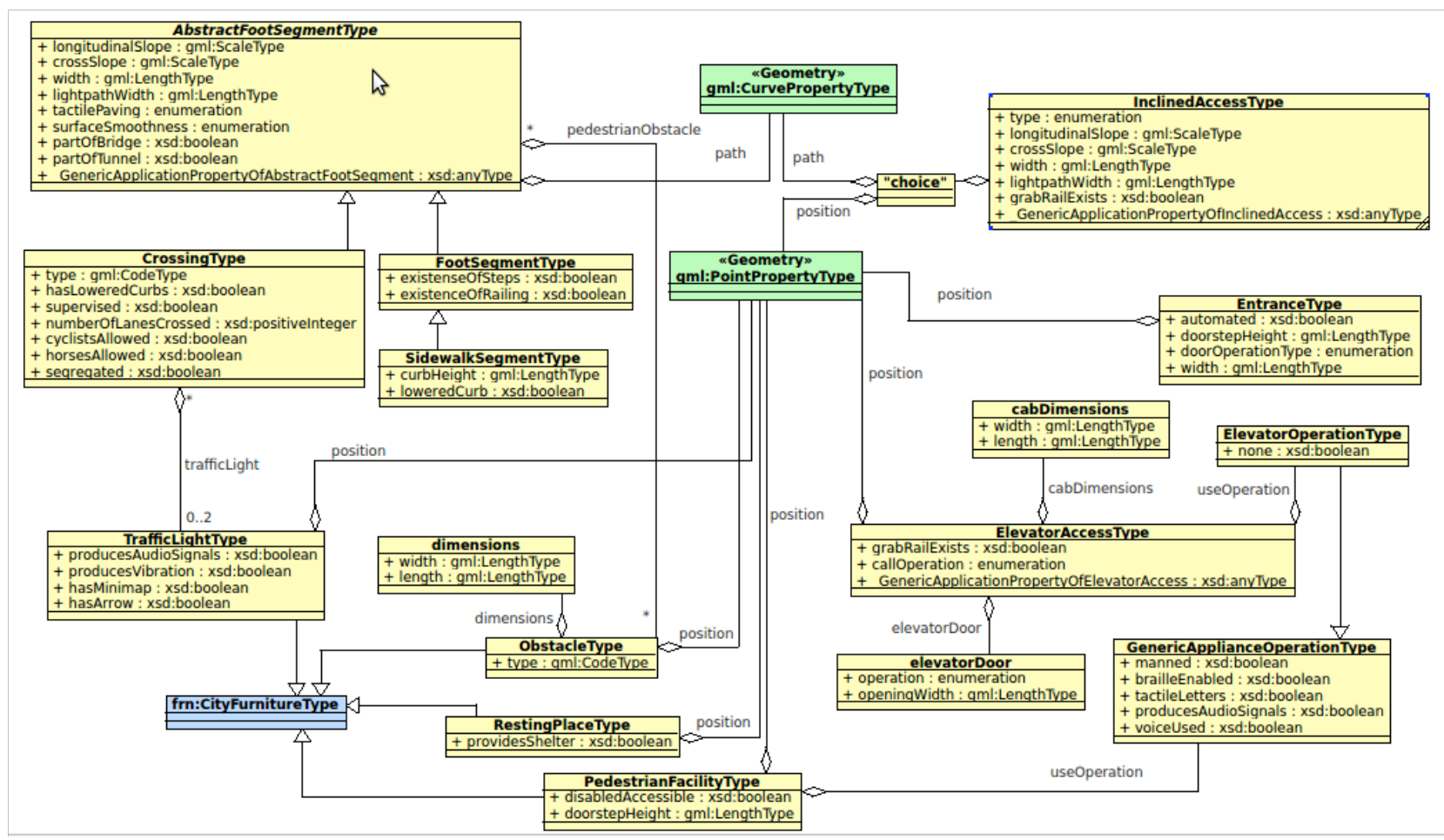

Figure 2: UML diagram of the i-SCOPE inclusive routing ADE

model an interoperable container for the services information and 2) how to provide in an effective mode the $3 \mathrm{D}$ model to the final services users.

The first item will be managed through the Application Domain Extensions (ADEs), for each services domain a specific ADE will be defined, conceptual model and implemented. The web provisioning and visualization of $3 \mathrm{D}$ city model is a bottleneck on the huge diffusion of these technologies.

An overview of how i-SCOPE will deal the abovementioned issues will be shown in the next paragraphs.
Extensions to the CityGML data model applying to specific application fields can be implemented using the Application Domain Extensions (ADE). Generic objects/attributes allow extensions during runtime, but may cause arbitrarily and name conflicts because different user defined objects may have the same name.

i-SCOPE aims at providing a significant contribution to standards in the domain of smart city services, through contribution to extension and wider adoption of CityGML as key enabling open standards for 3D smart city services, in 
particular defining specific Application Domain Extension (ADE) in the i-SCOPE domains: inclusive routing, solar energy assessment and noise mapping.

For instance of the key services provided by i-SCOPE will be the inclusive routing. Into CityGML standard the transportation model provides the needed information to describe the transportation object however the specifications suggest using other encoding for routing purpose (Fig.2). The i-SCOPE project investigates the possibility to extend the core part of CityGML including routing capabilities. However considering: the already existing frameworks and standards for routing and the modularity of CityGML even using ADE, the final solution foreseen the extension of the standard developing and ADE and not changing the core objects. The routing functionalities will be ensured using existing platform (i.e. PGrouting) considering the barrier elements defined into disable routing ADE.

\subsection{System Architecture and CityGML visualizations}

Visualisation is a complex and important issue in 3D city model applications. Efficient visualisation of 3D city models in different levels of detail (LODs) is one of the pivotal technologies to support these applications and it is fundamental to visualise the urban environment in different scales, e.g. from overview scale like a region down to detailed scale like a building or even a room. Furthermore Internet has become a basic information infrastructure all over the world even for the deployment of new smart cities technologies. Therefore, it is necessary to develop methods to visualise $3 \mathrm{D}$ city models through the Internet.

Currently, many plugins of Internet browsers have been developed to display 3D scenes such as Adobe Flash, Microsoft Silverlight and Java3D etc. But sometimes 3D content cannot be

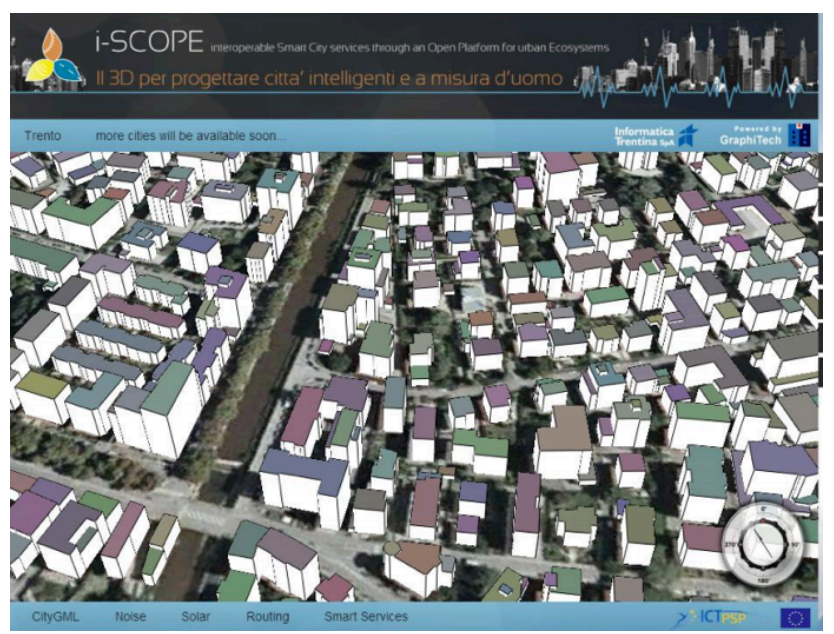

Figure 3 LoD1 model of Trento visualized into i_SCOPE 3D environment

opened since the user did not install the right plugins (Fassi and Parri, 2012).

Challenges of $3 \mathrm{D}$ city model visualisation include how to create the 3D scenes for multiple platforms through Internet and how to automatically generate the multiple representations for different scales (fig.1).

Streaming three-dimensional geographic data is a relatively new topic and no standard has yet been taken hold, OCG proposed three different services able to stream three- dimensional data to a client. One can be considered the extension of the other, differing in the amount of computational power required by the client and the server.
I-SCOPE project implements the possible services to stream the data to the client considering two different features: a) obtain optimum performance and very short response time avoiding the complex queries that can be made with the WFS; b) transmit to the client the geometries plus the semantic information in a single stream.

The way to obtain this kind of results is to stream data directly in the CityGML format. The method consists in a downloading service, which provides to the client the CityGML data following a classic tile-based approach. To achieve these objectives first a tiled based CityGML is generated in a structured tree and second, a progressive visualization of the model into client dynamically asking the server for new visualization or level of detail when the user changes the viewpoint or zoom level in the $3 \mathrm{D}$ viewer. A common tile grid is defined, and well knows both on server and client side. All the needed CityGML data is stored on the file system of the
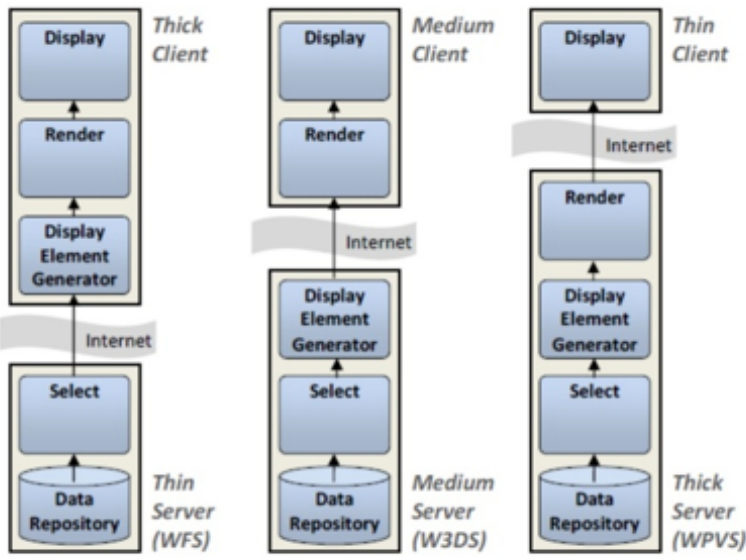

Figure 4 WFS, W3DS and WPVS (deprecated) compared. Source: Draft for Candidate OpenGIS ${ }^{\circledR}$ Web 3D Service Interface Standard. OGC document 09-104r1.

server, avoiding the access to a database that can slow down the entire operation. The data is stored in tiles according the selected grid and all the files are compressed to use less bandwidth in the transmission phase. Due to the verbose format of the CityGML an incredible compression rate can be achieved, reducing the size of the dataset up to the $90 \%$ if no textures are included. The stored compressed files must follow the name schema pre-defined and corresponding to the tile grid name schema. This approach can be classified into the Thick Client/Thin Server described into fig. 4, but avoiding the issues related to the complex operation on server side typical of WFS (fig.5).

Within the iSCOPE project the client has been developed on top of Nasa World Wind java SDK. Thanks to this approach many useful features can be implemented in parallel: different services like the WMS, WFS and the proposed approach can run concurrently allowing a great flexibility of the entire system.

\section{SMART CITIES SERVICES}

In this section will be presented an overview of the smart services provided by the i-SCOPE platform and based on 3D CityGML model. Indeed considering i-SCOPE perspective CityGML is the base container in on which a series of smart city services will be provided. The services foreseen by the platform are three: 
Service for accurate assessment of solar energy potential at building level.

Improved inclusion and personal mobility of aging and diversely able citizens through an accurate city-level disable-friendly personal routing service which accounts for de-tailed urban layout, features and barriers.

Environmental monitoring through a real-time environmental noise mapping service-leveraging citizen's involvement will who act as distributed sensors city-wide measur-ing noise levels through their mobile phones.

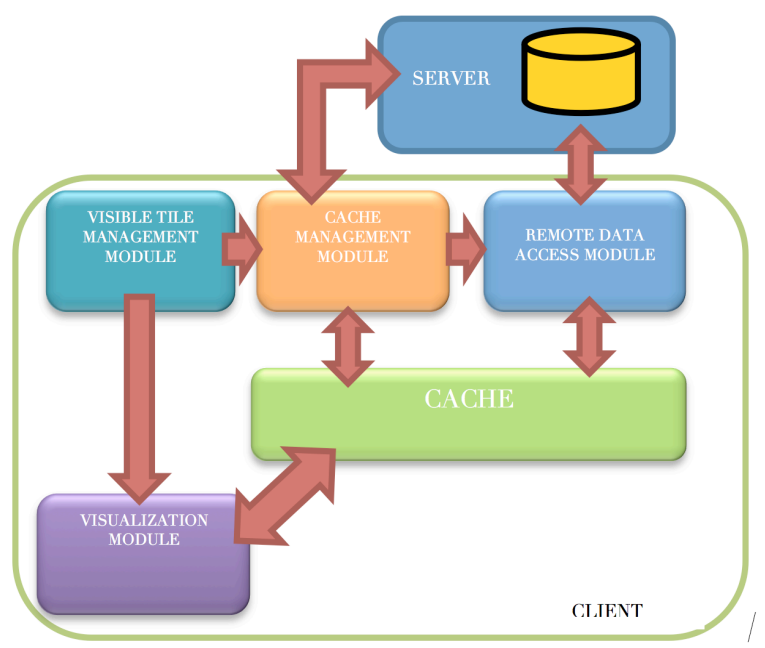

Figure 5 System architecture of the proposed approach

Several methodological and technological matters are related to the set up of the services:

- Definition of the ADEs in according to the services functionality requirements;

- $\quad$ To Develop the process and interfaces between different components in order to provide services;

- Design a web user interface for the citizen in order to use the services in a very easily way.

These services will be piloted and validated, within a number of EU cities, which will be actively en-gaged throughout the project lifecycle and covering different aspects of the urban environment they would demonstrate as a platform smart-city platform could be more effective if the services are provided on a common base infrastructure.

\subsection{Solar potential assessment}

Today, different methodologies for the estimation of PV potential at an urban scale are pro-posed. Accurate 3D modelling is very important to accurate photovoltaic system simulation however advanced feature as including mutual shading of buildings in urban settings is still quite weak (Alam, 2010).

From a computational point of view i-SCOPE provides a service for sun irradiation calculation and estimation of Photovoltaic potential. In order to achieve this the "r_SUN" function - module of GRASS will be customized and integrated in the platform's functionality by creating a WPS interface. The CityGML building models through an algorithm based on Raycasting is rasterized and used as input for the solar irradiation calculation. The irradiation values are insert into the ADE for each corresponded roof elements.
The user access the i-SCOPE web services platform and setting up some parameters such as: panels area (percentace of entire roof), efficiency, orientation (in flat roof case) can estimate the solar potential of each roof in a given period.

\subsection{Crowd Source noise services}

Currently over $50 \%$ of the world's population lives in urban areas. Being continuously sur-rounded by traffic jams, construction sites and urban events, city dwellers are typically exposed to a considerable amount of noise. In order to assess the distribution of this noise pollution (in time and space) the iScope project proposes the integration of participatory techniques for monitoring pollution by means of NoiseTube. NoiseTube has been designed to facilitate sound measuring at any place and any time through a mobile app that exploits basic smartphone functionalities, namely microphone, wireless connectivity and localisation through GPS. Through these three components NoiseTube transforms already ubiquitous smartphones into highly port-able, accessible sound measurement devices, enabling all citizens to measure ambient sound levels whenever and wherever they please.

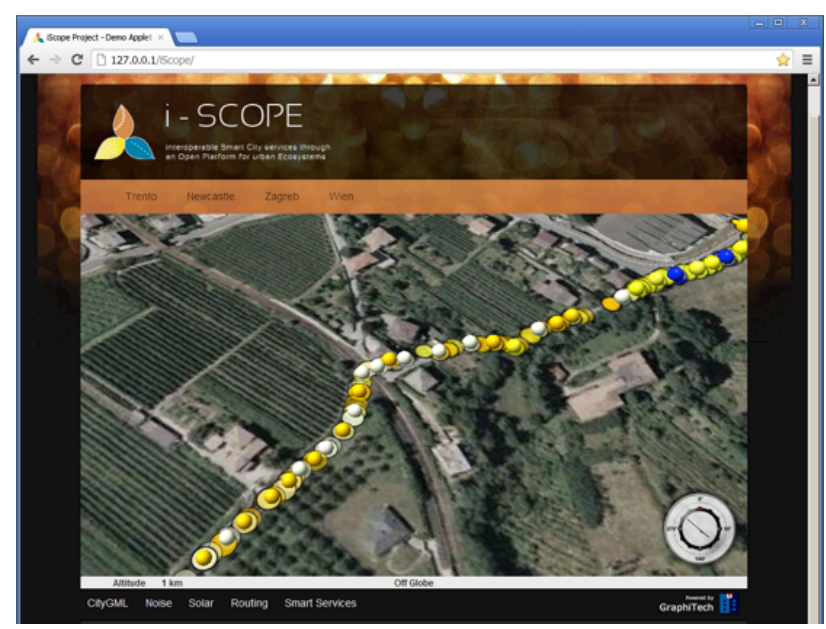

Figure 6 NoiseTube data acquired visualized into i-SCOPE web portal

Next to the mobile app a second pillar of our participatory approach is the NoiseTube website which collects all user measurements and visualises them on i-SCOPE platform (fig. 6).

The successful collection of information by masses of volunteering individuals enabled by Web technology (otherwise referred to as Web 2.0) does not halt before the realm of geographic in-formation. Even more remarkable in the given context, several projects concentrating solely on the collection of geographic information have formed. Goodchild (2007) gives an overview of these global collaborations and calls the phenomenon Voluntary Geographic Information (VGI).

Given enough measurements for a particular area, we can construct noise maps of comparable quality to those produced by governments today, which are of a very different kind. Indeed, pollution maps are typically created through computer simulations based on general statistics, such as the average number of cars in the city. They are backed up only by limited amounts of sound measurements because current measuring methods are expensive and thus not very scalable. The resulting maps give an average but not at all a complete view on the situation, entirely missing local variations due to street works, neighbour noise \&etc. The collected information are made available within the i-SCOPE platform so the combined 
visualization of noise data and 3D CityModel became a powerful instrument to understand the city "noise feeling".

\section{CONCLUSION}

This paper reports the results of the first years work of EU project i-SCOPE which based on interoperable 3D UIMs CityGML, would to deliver an open platform on top of which it develops, within different domains, various 'smart city' services.

In terms of products and services i-SCOPE delivers an open source toolkit for 3D smart city services that will be deployed and made available through a 3D smart city services portal.

Furthermore i-SCOPE aims at providing a significant contribution to standards in the domain of smart city services, through contribution to extension and wider adoption of CityGML as key enabling open standard for 3D smart city services.

Many challenges in terms of encoding standards, efficient visualization of 3D geometries, data and services generation and providing and privacy model will be deal during this phase.

For whom concern the standardization one specific ADE for a particular service is on going definition using UML specification.

The needed information collected by pilot partners are used to automatically generate the 3D cityModel of buildings into LoD1 and LoD2. Furthermore information for disabled routing are not already available are collected using the OSM model and enriched according the specific ADEs using the available tools. At the same way campaign to acquire large scale of noise measurements using noiseTube application are started.

The way to exchange 3D information between i-SCOPE server and web-client has been defined considering a percalculated tiling approach in order to improve the performance of the system.

The security and privacy model will be trialled and optimised in both the i-SCOPE and i-Tour projects and in a number of ITS trials from early 2013 pending its full scale launch in 2016 (planned) for C-ITS and slightly earlier in the pilots at the heart of i-SCOPE.

Many issues have to be solved to obtain an effective platform to provide smart-cities services into a 3D city model environment, however the good results obtained demonstrate that combining technologies for 3D visualization, open standards and VGI it is possible to deploy a smart-city spatial data infrastructure.

\section{ACKNOWLEDGMENTS}

The project I-SCOPE has received funding from the EC, and it has been co-funded by the CIP-Pilot actions as part of the Competitiveness and innovation Framework Programme. The author is solely responsible this work which does not represent the opinion of the EC. The EC is not responsible for any use that might be made of information contained in this paper.

\section{REFERENCES} $\begin{array}{lcl}\text { 3DCityDB, } & (2011): & \text { http://opportunity.bv.tu- } \\ \text { berlin.de/software/projects/3dcitydb. } & \end{array}$
Alam N., 2010. Shadow effect on 3D City Modelling for Photovoltaic Cells. PhD Research Proposal, GISr Re-port; p. 56.

Al-Hader, Mahmoud, et al., 2009 "SOA of smart city geospatial management." Computer Modeling and Simu-lation. EMS'09. Third UKSim European Symposium on. IEEE, 2009.

Cisco, (2005), Dubai: The Smart City:

http://www.cisco.com/web/learning/le21/le34/downloads/689/n obel/2005/docs/Abdulhakim_Malik.pdf

Desmet, K., and Esteban Rossi-H., 2012 "Analyzing Urban Systems: Have Mega-Cities Become Too Large?.".

Cook Diane, Sajal Das, 2005 "Smart Environments: Technologies, Protocols, and Applications”, Wiley-Interscience.

Döllner, J., Kolbe, T. H., Liecke, F., Sgouros, T. And Teichmann, K. 2006. The virtual 3D city model of Berlin managing, integrating and communicating complex urban information. In 25th International Symposium on Urban Data Management UDMS.

Fassi F., Parri S., 2012 Complex Architecture in 3D: From Survey to Web. International Journal Of Heritage In The Digital Era. 01/2012; 1:379-398.

Goodchild, M.F., 2007. Citizens as sensors: the world of volunteered geography, In: GeoJournal, Vol.69. Issue 4, pp. 211-221.

IBM, (2009), Smarter Cities: New York 2009:

http://www.ibm.com/smarterplanet/us/en/smarter_cities/article/ newyork2009.html

Ishida T., Isbister K., 2000. "Digital Cities: Technologies, Experiences, and Future Perspectives”, Springer-Verlag. Ishida, "Digital city kyoto." Communications of the ACM 45.7 (2002): 76-81.

McCarra, Darren. "Google Maps 3D building." (2012):

http://.sociable.co/technology/google-maps-next-dimensionrevealed-with-fully-automated-3d-cityscapes/

McCarra, Darren. "Apple will debut incredible in-house 3D Maps app next month." (2012):

http://sociable.co/mobile/apple-will-debut-incredible-in-house3d-maps-app-next-month/

OPENGIS. 2008. City Geography Markup Language (CityGML) Encoding Standard. Document 08-007r1, version 1.0 .0

Prandi F., De Amicis R, Conti G., Debiasi A., 2012 Use of OGC web standard for a spatio-temporal enabled SDI for civil protection. Proceedings of the 17 th International Conference on 3D Web Technology, 105-111

Roche, S., and Abbas R. 2012 "Sensing places' life to make city smarter." Proceedings of the ACM SIGKDD International Workshop on Urban Computing. 